\title{
Contextual risk factors for the common mental disorders in Britain: a multilevel investigation of the effects of place
}

\author{
S Weich, L Twigg, G Holt, G Lewis, K Jones
}

J Epidemiol Community Health 2003;57:616-621

See end of article for authors' affiliations

\section{Correspondence to:}

Dr S Weich, Department of

Psychiatry and Behavioural Sciences, Royal Free and

University College Medical

School, Royal Free

Campus, Rowland Hill

Street, London NW3 2PF

UK; s.weich@rfc.ucl.ac.uk

Accepted for publication 8 January 2003

\begin{abstract}
Study objective: To test hypotheses about associations between area level exposures and the prevalence of the most common mental disorders (CMD) in Britain. A statistically significant urban-rural gradient was predicted, but not a socioeconomic gradient, in the prevalence of CMD after adjusting for characteristics of individual respondents. The study tested the hypothesis that the effects of area level exposures would be greatest among those not in paid employment.

Design: Cross sectional survey, analysed using multilevel logistic and linear regression. CMD were assessed using the General Health Questionnaire (GHQ). Electoral wards were characterised using the Carstairs index, the Office of National Statistics (ONS) Classification of Wards, and population density.

Setting: England, Wales, and Scotland.

Participants: Nearly 9000 adults aged 16-74 living in 4904 private households, nested in 642 electoral wards.

Main results: Little evidence was found of statistically significant variance in the prevalence of CMD between wards, which ranged from $18.8 \%$ to $29.5 \%$ (variance $0.035, \mathrm{SE} 0.026)(p=0.11)$. Associations between CMD and characteristics of wards, such as the Carstairs index, only reached statistical significance among those who were economically inactive (adjusted odds ratio for top $v$ bottom Carstairs score quintile $1.58,95 \% \mathrm{Cl} 1.08$ to 2.31 ) ( $p<0.05)$

Conclusions: There may be multiple pathways linking socioeconomic inequalities and ill health. The effects of place of residence on mental health are greatest among those who are economically inactive and hence more likely to spend the time at home.
\end{abstract}

$\mathrm{D}$ espite evidence of causal associations between individual level socioeconomic deprivation and higher rates of psychiatric morbidity, ${ }^{2}$ comparatively little is known about how the places where people live affect their mental health. ${ }^{3}$ Studies of geographical inequalities in rates of the most common mental disorders (CMD), anxiety and depression, have provided inconsistent but intriguing findings. In the UK, two early studies found no evidence of statistically significant variation in the prevalence of CMD between regions (which have populations comprising several million people), after adjusting for the characteristics of individual respondents. ${ }^{45} \mathrm{~A}$ more recent study reported similar findings at electoral ward level (average population 5500), in which areas were classified using a standard census based compositional measure of socioeconomic deprivation. ${ }^{6}$ Similar findings have also been reported in Amsterdam, at three levels: borough (average population 33000 ), neighbourhood (average population 8000), and postcode sector (average population 9500). ${ }^{78}$ By contrast, a household survey in Illinois found a small but statistically significant association between depressive symptoms and deprivation at the level of US census tracts (average population 4000), after controlling for individual level risk factors. ${ }^{9}$

In contrast with these mainly negative findings, a number of British studies have found a higher prevalence of CMD among people living in urban areas, compared with rural or suburban areas. ${ }^{10-12}$ At least one study found that suicide rates were also higher in urban than in rural areas in Britain, ${ }^{13}$ although rates may vary with gender and both area level and individual socioeconomic factors, particularly unemployment. ${ }^{14}{ }^{15}$ However, studies in New Zealand ${ }^{16}$ and the $\mathrm{USA}^{17}$ found little evidence of an urban/rural gradient in the prevalence of CMD. These inconsistencies may be partly attributable to differences in methodology, including differ- ences in the definition of "urban" areas. While most studies have quantified urbanicity according to population density, others have used more subjective, impressionistic definitions ${ }^{12}$ on the grounds that ward level population density may not reflect smaller scale contextual variation. ${ }^{11}{ }^{12}$

Advances in multilevel modelling enable potential risk factors for a given outcome to be studied at more than one level simultaneously, along with cross level interactions. ${ }^{18-21}$ However, most previous studies have failed to take into account variability between households, resulting in over-estimates of variance at higher levels. ${ }^{156922}$ Associations between health outcomes and area level exposures tend to diminish with the number of individual level confounders included in models, ${ }^{3}$ suggesting that some of the observed area level effect may reflect variation at the individual level. Even if there is little residual area level variation in rates of CMD once individual characteristics have been taken into account, it does not mean that complex heterogeneity is absent. Different relations for different types of people across place may exist, but these interactions can only be fully explored if models are specified correctly, and designed to test theoretically informed hypotheses. ${ }^{9}{ }^{20}$ For instance, one study found that the effects of household level exposures on self rated health varied with economic activity. ${ }^{23}$

We sought to address these limitations by analysing data on persons in UK electoral wards using measures of place that reflect both socioeconomic composition and urbanicity, and by extending the notion of "place" to include the household. The main aim of this study was to quantify independent

Abbreviations: CMD, common mental disorders; BHPS, British Household Panel Survery; GHQ, General Health Questionnaire 
associations between the prevalence of depression and (a) area level indices of socioeconomic deprivation, and (b) urbanicity and population density, after adjusting for individual socioeconomic status. We set out to test the hypotheses that (1) the prevalence of CMD in Britain is higher to a statistically significant degree among people living in urban areas, compared with rural and suburban areas, after adjusting for the socioeconomic status of individual respondents; and (2) the prevalence of CMD will be higher to a statistically significant degree among people living in areas with the highest levels of socioeconomic deprivation, before but not after adjusting for the socioeconomic status of individual respondents. A further aim was to test for interactions between exposures at different levels, and to explore variations in the prevalence of CMD within urban and rural areas. On the grounds that those who are economically inactive are likely to spend more time at home, we also tested the hypothesis that (3) the effects of area level exposures would be greatest among those not in paid employment.

\section{METHODS}

Data were derived from the first wave of the British Household Panel Survey (BHPS) that was carried out in autumn 1991. ${ }^{22}{ }^{24}$ The BHPS is an annual survey of a representative sample of people in private households in England, Wales, and Scotland. Households were selected for the BHPS using a two stage, implicitly stratified clustered probability design, with postcode sectors as primary sampling units. ${ }^{24}$ The population of postcode sectors was first ordered into 18 regions ( 16 standard regions in England, distinguishing former metropolitan counties and inner and outer London in England, plus Wales and Scotland), resulting in a sample broadly representative of regional populations. Interviews were conducted with all members of selected households aged 16 and over. Individual BHPS participants aged 16-75 who completed the General Health Questionnaire (see below) were included in this analysis. The BHPS investigators complied, in full, with the ethical guidelines of the Social Research Association. ${ }^{24}$

\section{Assessment of common mental disorders}

CMD were assessed using the self administered 12 item General Health Questionnaire (GHQ), which has been widely validated against standardised clinical interviews. ${ }^{25}$ We followed previous studies in treating the $\mathrm{CMD}$ as a single dimension, ${ }^{25-27}$ whereby GHQ score represents the probability of a person being a psychiatric case. ${ }^{25}$ Each item on the GHQ was scored in two ways: either using a four point Likert scale (range 0 to 3 ), or as present or absent. Using the former method produces scores that approximate a normal distribution, while those scoring 3 or more (out of 12) by the second method were classified as cases. ${ }^{1222528}$ We conducted all analyses using both scoring systems. The findings did not markedly differ according to whether the outcome was treated as a dichotomous or continuous variable and results here have only been reported for the presence or absence of a CMD "case".

\section{Area level exposures}

The choice of area level exposures was limited by the absence of any validated contextual measures, and a dearth of evidence about which of the large number of compositional measures were likely to be associated with the prevalence of the common mental disorders. Electoral wards (mean population 5222, SD 3899) are currently the smallest geographical area at which BHPS data are available. The BHPS investigators and authors agreed a method for matching respondents and characteristics of electoral wards, without disclosure of information that might permit identification of respondents. To preserve respondents' anonymity, we were restricted to a total of three linked, ward level indices, only one of which could be analysed at a time.
We therefore chose, a priori, to use (a) the Carstairs index of socioeconomic deprivation, ${ }^{29}$ (b) the Office of National Statistics (ONS) classification of wards into 14 groups, ${ }^{30}$ and (c) a measure of population density given as the number of 25-64 year olds per square kilometre. The first two of these were based on data collected in the 1991 census and the density measure was derived from re-worked 1991 census data that attempted to adjust for the census undercount. ${ }^{31}$ The Carstairs index ${ }^{29}$ is based on $\mathrm{Z}$ scores of four person level (compositional) variables for each ward: male unemployment, households with no car, overcrowding (over one person per room), and head of household in registrar general's social class IV or V. Both Carstairs scores and population densities were rounded to integer values. The BHPS investigators truncated the tails of the distribution of Carstairs scores, to protect the identities of survey respondents.

The ONS classification of wards ${ }^{30}$ comprises 14 principal groups and 43 clusters of electoral wards, based on their demographic and socioeconomic composition. More than 30 census variables were used to generate this classification, including age, ethnicity, household composition, education, housing tenure, employment status, and the proportion of residents working in different occupations. Although no direct measures of the physical environment were used, proportions of respondents living in terraced and purpose built housing were included. Groups and clusters were derived by ONS using Ward's method, a two stage cluster analysis technique, followed by a k-means procedure with iteration at cluster level to ensure wards were assigned to the cluster with the smallest dissimilarity between it and the cluster centroid..$^{32}{ }^{33}$ The final classification was designed to ensure that clusters were homogenous, and sufficiently populous to permit the study of geographical patterns. Particular attention was paid to the face validity of clusters, so that they might be recognisable and meaningful to users. Groups and clusters were given names by the originators of classification "for ease of reference, based on the general characteristics of cluster members . . sometimes combined with [their] geographic attributes". ${ }^{33}$ These names were therefore intended as shorthand, rather than precise descriptions. A full list of groups and clusters, and portraits of each, are available elsewhere. ${ }^{32} 33$

Associations with the prevalence of CMD were estimated in two ways: (a) for each of the 14 groups, and (b) for "rural areas" versus the rest. Three groups, namely "rural fringe", "rural area", and "prosperous area" groups were aggregated to produce a single dummy variable representing "ONS rural grouping". This 'rural' aggregation was done a priori, on the basis of the geographical distribution of the groups in question, the identities of the clusters included within each group,$^{30}$ and population densities. As table 1 shows, the three rural groups were those with the lowest population densities. The mean population density in the "ONS rural grouping" was lower than that in the remaining 10 ONS groups, to a statistically significant degree (difference between means 1242.5, 95\% CI 1179.1 to 1305.9$)(\mathrm{p}<0.001)$.

\section{Individual and household exposures}

Individual and household level confounders were chosen both to mirror the indices used to construct the Carstairs index, the area level measure of socioeconomic status used in this study, and using existing evidence concerning known risk factors for $\mathrm{CMD}^{1}{ }^{22}$ At the individual level, age, sex, marital status, ethnicity, employment status, and number of current physical health problems were included. Employment status was classified using three categories: employed (in paid employment), unemployed (available for and seeking paid employment), and economically inactive (not currently available for employment). The last group includes the retired, full time students, those unable to work on health grounds, and full time carers.

Potential household level confounders were: social class of the head of household, structural housing problems (any major 
Table 1 Numbers of respondents (n), prevalence of common mental disorders (\% cases CMD), and mean population densities (standard deviation) (persons aged 25-64 per square kilometre), for each of the 14 groups identified in the Office of National Statistics (ONS) Classification of Wards

\begin{tabular}{llll}
\hline Group & $\mathrm{n}$ & $\begin{array}{l}\text { Mean density } \\
\text { (SD) }\end{array}$ & $\begin{array}{l}\text { \% Cases } \\
\text { CMD }\end{array}$ \\
\hline Metropolitan professionals & 310 & $2809(1121)$ & 29.5 \\
Deprived city areas & 568 & $3954(1500)$ & 29.4 \\
Deprived industrial areas & 454 & $1891(882)$ & 27.7 \\
Low status owner occupiers & 508 & $1630(1089)$ & 26.5 \\
Industrial areas & 941 & $1271(710)$ & 25.8 \\
Mature populations & 651 & $1014(679)$ & 25.1 \\
Inner city estates & 148 & $3128(2293)$ & 25.0 \\
Middle Britain & 1400 & $1077(724)$ & 25.0 \\
Transient population & 32 & $1258(643)$ & 24.7 \\
Suburbia & 1318 & $1457(826)$ & 24.6 \\
Rural fringes & 716 & $457(606)$ & 24.1 \\
Established owner occupiers & 1054 & $752(691)$ & 21.6 \\
Prosperous areas & 564 & $217(301)$ & 20.6 \\
Rural areas & 269 & $23(19)$ & 18.8 \\
\hline
\end{tabular}

problem, or two or more minor problems from a list comprising damp, condensation, leaking roof and rot in $w_{0 o d^{22}}$ ), low income (household income below half the median income for the sample), household access to a car, and overcrowding (more than two household members per bedroom).

\section{Statistical analysis}

Multilevel approaches offer substantive improvements over traditional regression methods, particularly for hierarchical datasets such as the BHPS. ${ }^{34}$ All analyses were undertaken using MlwiN, ${ }^{35}$ which takes account of the clustering of persons in households and households in wards when estimating standard errors. ${ }^{18-21}$ Individuals (level 1) were nested within households (level 2), within electoral wards (level 3). Multilevel analyses were undertaken using a logit link function and assumed non-constant, between individual variance based on a Bernoulli distribution. ${ }^{19}$ In the logistic models, parameters were estimated using second order Taylor expansion with predictive quasi-likelihood (PQL). This estimation procedure is considered superior to first or second order marginal quasi-likelihood (MQL) when clusters, such as households, are small (see Goldstein, ${ }^{19}$ chapter 7). While the quality of parameter estimates may be more accurate still for small clusters using Markov chain Monte Carlo (MCMC) methods, ${ }^{36}$ this method is computationally intensive and was therefore used to check our main findings. Differences between methods were expected to be more pronounced for estimates of variance at the household than at the ward level. Differences using second order PQL and MCMC methods were noted where appropriate.

Base models were used to estimate associations with each contextual (level 3) variable prior to adjusting for individual and household level characteristics. Each model was then adjusted for individual and household level variables. Given the variable number of respondents per ward (range 1 to 60), sensitivity analyses were undertaken excluding (a) wards with only one respondent, and (b) wards with five or fewer respondents. Statistical significance of individual fixed estimates was tested using a single Wald test against a $\chi^{2}$ distribution. While approximate Wald tests can be used to assess the higher level variances, problems are encountered because of the distribution of the parameter estimates when variances are close to zero (negative variances cannot exist). Therefore the 95\% interval estimates (the "credible interval") derived from MCMC procedures are also reported for the random parameters of the models. Multivariate Wald tests were also used to assess blocks of interactions between individual and area level exposures.

\section{RESULTS}

After excluding "deadwood" addresses, $73.6 \%$ of households $(\mathrm{n}=5511)$ participated in the first wave of the BHPS. ${ }^{22}$ This resulted in a sample of 9522 people aged 16-74, of whom $94.3 \%(n=8978)$ completed the GHQ. Study respondents were nested within 4904 households. The total number of electoral wards represented was 642 , with a mean of 14 and a median of 11 respondents per ward (range 1 to 60 ). The prevalence of CMD was $24.7 \%$ among individuals, across all households and wards.

Table 1 shows the population density and unadjusted prevalence of CMD in each of the 14 ONS groups. Using the ONS classification of wards, the lowest prevalence of CMD (18.8\%) was found among respondents in rural areas (table 1 ). This rate was lower to a statistically significant degree than that observed in 7 of the remaining 13 groups, namely Metropolitan Professionals, Deprived City Areas, Deprived Industrial Areas, Lower Status Owner Occupiers, Industrial Areas, Mature Populations, and Middle Britain.

Table 2 summarises the results of the multilevel models investigating contextual effects. We found evidence of interactions between characteristics of wards and employment status, in keeping with our hypothesis that associations between characteristics of places and CMD would be greatest for those most likely to spend more time at home (table 2). However, in analyses based on the full dataset, the interaction with employment status was only statistically significant for Carstairs score in the unadjusted model $\left(\chi^{2}=16.063, \mathrm{df}=8\right.$, $\mathrm{p}=0.04$ ). Associations between Carstairs score, rurality (“ONS rural grouping" versus the rest), population density, and CMD only reached statistical significance among the economically inactive.

Random variances were estimated at the household and area levels before and after including specific explanatory variables in the fixed part of our models. In the null model, before specifying any characteristics of individuals, households or wards but taking account of the nested structure of the dataset, we found statistically significant variance in the prevalence of CMD between households (variance 0.565, SE 0.077) (Wald 53.84, $\mathrm{p}<0.001$ ) but not between wards (variance 0.035, SE 0.026) (Wald 2.53, p=0.11). Slightly different findings were obtained using MCMC methods, particularly at the household level where variance was estimated to be 0.794 (credible interval 0.54 to 1.057 with 100000 chains). The ward level variance using MCMC methods was 0.032 (credible interval 0.001 to 0.098). The household level variance was little affected by specifying characteristics of wards, and remained statistically significant (although attenuated) after adjusting for individual and household level risk factors, irrespective of statistical method. The (non-statistically significant) ward level variance was reduced substantially by specifying ward level characteristics (particularly ONS groups), and approached zero in all cases on adjusting for individual and household level risk factors.

\section{DISCUSSION}

In keeping with a number of previous studies, ${ }^{4-8}$ our findings indicate that there is comparatively little independent area level variance in the prevalence of the CMD. Like most ${ }^{6-8}$ (but not all ${ }^{9}$ ) previous studies, we found no evidence of a statistically significant overall association between the prevalence of CMD and socioeconomic deprivation, as measured by the Carstairs index. Our findings were particularly notable for demonstrating interactions between area level exposures and individual employment status, in keeping with at least one previous study. ${ }^{23}$ Associations between CMD and population 
Table 2 Unadjusted odds ratios (95\% confidence interval) for associations between the prevalence of common mental disorders and ward level socioeconomic deprivation (Carstairs index), rurality (using ONS Classification of Wards) and population density, respectively, by employment status, and adjusted for individual and household level confounders $\dagger$

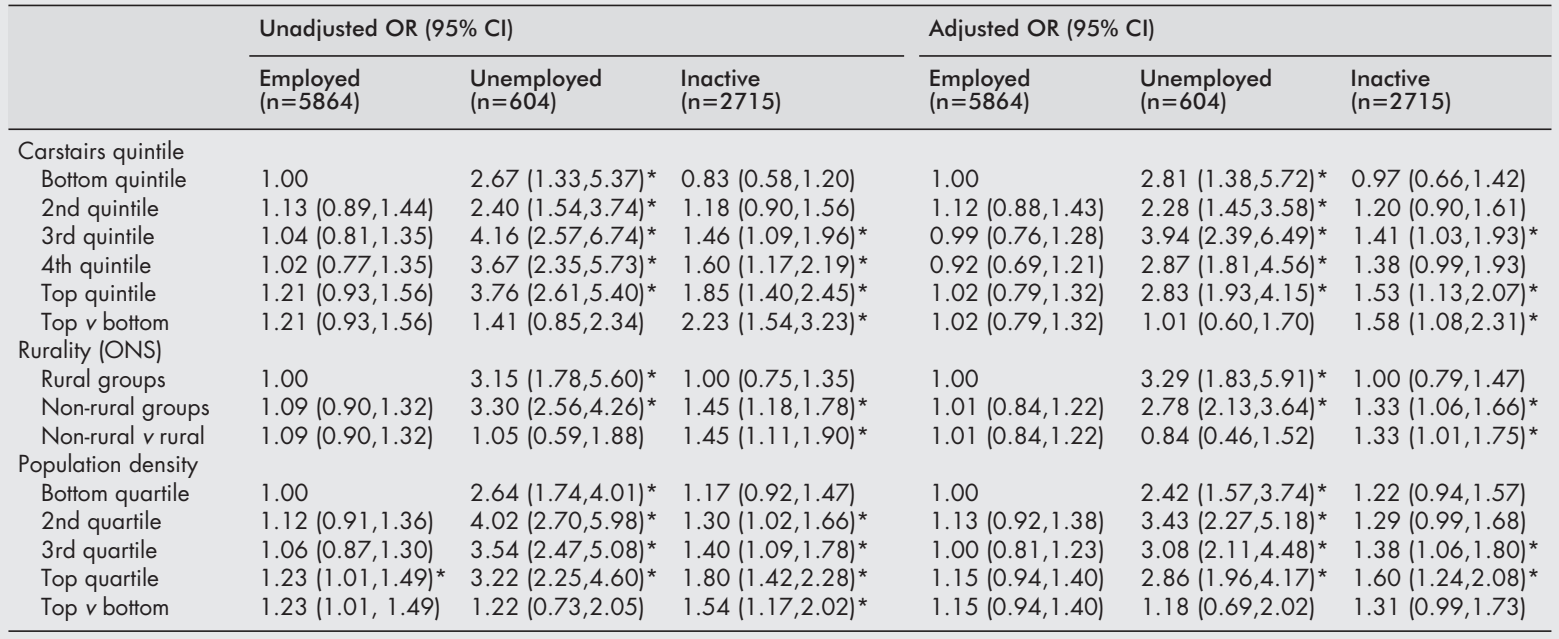

Odds ratios obtained from logistic models, in which parameters were estimated using second order Taylor expansion with predictive quasi-likelihood (PQL) (see Methods). †Age, sex, ethnicity, physical health problems, overcrowding, housing problems, low income, car access, and social class. ${ }^{*} p<0.05$.

density, Carstairs score (socioeconomic deprivation) and urbanicity were only statistically significant among the economically inactive.

\section{Measuring the CMD}

The study was limited by use of the GHQ rather than a standardised clinical interview. Associations between individual level risk factors, such as low income, and rates of CMD are generally larger in studies using a standardised interview. ${ }^{11}{ }^{37}$ As the GHQ is sensitive to recent change in psychological functioning, "false positives" might have included persons with mild or transient psychological disturbance, which should have biased associations towards the null. Although physical ill health also leads to "false positives", study findings were adjusted for the number of current physical health problems. As the GHQ is a measure of recent changes in mental state, some people with longstanding symptoms of anxiety and depression may be misclassified as non-cases. ${ }^{25}$ However, one previous study using the 12 item version of the GHQ in community settings found a total false negative rate of just $7 \% .{ }^{25}$ Although alternative scoring methods for the GHQ have been proposed to reduce this type of misclassification, none has been shown to be significantly superior to the methods used in this study. ${ }^{25} 38$

Those in lower occupational grades ${ }^{26}$ may under-report psychiatric symptoms on the GHQ compared with responses to a standardised clinical interview. This may have led to an under-estimate of the prevalence of CMD among socioeconomically deprived people, and may have contributed to the failure to find statistically significant associations with ward level deprivation. However, this effect was unlikely to have been of sufficient magnitude to affect our main findings, particularly as highly significant cross sectional associations have been found with socioeconomic deprivation at the individual level in this dataset. ${ }^{22}$

\section{Other limitations of the study}

This was a cross sectional study, precluding causal inference. While our findings do not contradict the view that deprived persons are clustered in deprived areas, the variance within wards in the prevalence of CMD was far greater than that between wards. While reverse causality and/or social selection are often invoked as explanations for geographical clustering, it is difficult to see how these processes might have contributed to our (mainly) negative findings with regard to area level variance. It is difficult to see how selective health related mobility, local authority housing allocations or systematic area based labour market disadvantage ${ }^{39}$ might lead to less variation in CMD between wards. These processes may however have contributed to the modest urban-rural gradient in the prevalence of CMD among the economically inactive.

Perhaps the most salient feature of any study of this nature is the size of area studied. "Neighbourhoods" remain difficult to define or delineate, but are unlikely to be coterminous with electoral wards. ${ }^{40}$ Wards may be too large and heterogeneous to permit the detection of contextual effects. It is notable that the one study to report positive findings quantified contextual deprivation at the smallest spatial scale. ${ }^{9}$ This is consistent with evidence of statistically significant associations between rates of CMD and specific features of the built environment assessed across small areas, after adjusting for characteristics of individual residents. ${ }^{91-44}$ The variance observed at household level in this study may have been partly attributable to exposures operating at an intermediate level between ward and household. This view is supported by the failure of household and individual level exposures to substantially reduce the household level (level 2) variance.

Like many studies, we were forced to rely on standard measures of "social deprivation" based on the aggregated socioeconomic characteristics of local residents. ${ }^{6-9}$ The high correlation between area level deprivation and individual socioeconomic status may explain the absence of a statistically significant association with the former, after controlling for the latter. Future research should be based on theoretically based compositional measures, or true "contextual" measures of place that cannot be reduced to the aggregated characteristics of local residents. ${ }^{35-47}$ Despite the large size of the study sample, the number of unemployed informants was comparatively small $(n=604)$. It is possible that the failure to find statistically significant associations between area level exposures and CMD in this group, or interactions between area level exposures and employment status, represents type II error.

\section{Conclusions}

Variance in the prevalence of the CMD between electoral wards in the UK is modest, particularly when compared with 


\section{Key points}

- It is still not known whether, or how, the places where people live affect their mental health, independent of their individual or household circumstances.

- Multilevel statistical methods permit the analysis of variance at different levels simultaneously, and allow for the testing of cross level interactions. Despite this, previous studies have often failed to model variance at the household level.

- Associations between ward level exposures and the prevalence of the common mental disorders (CMD), anxiety and depression, varied with (individual) employment status. These associations were greatest among the economically inactive, who are likely to spend the most time at home.

that between individuals, and between households. Associations between ward level socioeconomic deprivation, urbanicity, and population density, and the prevalence of CMD varied with individual employment status, and were strongest among the economically inactive. These findings support the view that the causal pathways linking socioeconomic inequality and health may vary according to individual circumstances. Our results are consistent with previous research suggesting that features of households (or areas) may be most salient for those who are not in work, and who spend the most time at home. ${ }^{23}$

Further research is needed to understand why the effects of place seem to vary with individual employment status. Research is also needed to address prior questions, namely how and why the most deprived individuals come to be living in (or fail to "escape" from) socioeconomically deprived areas, which also tend to be the most densely populated and the most urban. ${ }^{48}{ }^{49}$ Investigating the effects and determinants of adverse physical $^{41} 4450$ and psychosocial environments ${ }^{51-53}$ are therefore a priority. Until we understand these processes, it will be difficult to design effective area level interventions to reduce inequalities in mental health.

\section{ACKNOWLEDGEMENTS}

We are indebted to Professor Nick Buck of the Institute for Social and Economic Research for his help in obtaining and managing the BHPS dataset with ward level indices. The data (and tabulations) used in this (publication) were made available through the UK Data Archive. The data were originally collected by the ESRC Research Centre on Micro-social Change at the University of Essex, now incorporated within the Institute for Social and Economic Research. Neither the original collectors of the data nor the Archive bear any responsibility for the analyses or interpretations presented here.

\section{Authors' affiliations}

S Weich, Department of Psychiatry and Behavioural Sciences, Royal Free and University College Medical School, London, UK

L Twigg, G Holt, Institute for the Geography of Health, Department of Geography, University of Portsmouth, Portsmouth, UK

G Lewis, Division of Psychiatry, University of Bristol, Bristol, UK

K Jones, School of Geographical Sciences, University of Bristol

Funding: this study was funded by the Wellcome Trust.

Conflicts of interest: none declared.

\section{REFERENCES}

1 Weich S, Lewis G. Poverty, unemployment and common mental disorders: population-based cohort study. BM 1998;317:115-19.

2 Lewis G, Bebbington P, Brugha T, et al. Socioeconomic status, standard of living, and neurotic disorder. Lancet 1998;352:605-9.

3 Pickett KE, Pearl M. Multilevel analyses of neighbourhood socioeconomic context and health outcomes: a critical review. $J$ Epidemiol Community Health 2001;55:111-22.

4 Lewis G, Booth M. Regional differences in mental health in Great Britain. J Epidemiol Community Health 1992;46:608-11.

5 Duncan C, Jones K, Moon G. Psychiatric morbidity: a multilevel approach to regional variation in the UK. J Epidemiol Community Health 1995;49:290-5
6 McCulloch A. Ward-level deprivation and individual social and economic outcomes in the British Household Panel Study. Environment and Planning A 2001;33:667-84.

7 Reijneveld SA, Schene AH. Higher prevalence of mental disorders in socioeconomically deprived urban areas in the Netherlands: community or personal disadvantage? J Epidemiol Community Health 1998;52:2-7.

8 Reijneveld SA, Verheij RA, de Bakker DH. The impact of area deprivation on differences in health: does the choice of the geographica classification matter? J Epidemiol Community Health 2000;54:306-13.

9 Ross CE. Neighborhood disadvantage and adult depression. J Soc Health Behav 2000;41:177-87.

10 Lewis G, Booth M. Are cities bad for your mental health? Psychol Med 1994;24:913-16.

11 Meltzer H, Gill B, Petticrew M. OPCS Surveys of Psychiatric Morbidity in Great Britain. Report No 1. The prevalence of psychiatric morbidity among adults aged 16-64 living in private households in Great Britain. London: HMSO, 1995.

12 Paykel ES, Abbott R, Jenkins R, et al. Urban-rural mental health differences in Great Britain: findings from the National Morbidity Survey Psychol Med 2000;30:269-80

13 Saunderson T, Haynes R, Langford IH. Urban-rural variations in suicides and undetermined deaths in England and Wales. J Public Health Med 1998:20:261-7

14 Kelly S, Charlton J, Jenkins R. Suicide deaths in England and Wales, 1982-92: the contribution of occupation and geography. Popul Trends 1995;80: 16-25.

15 Saunderson TR, Langford IH. A study of the geographical distribution of suicide rates in England and Wales 1989-92 using empirical Bayes estimates. Soc Sci Med 1996;43:489-502.

16 Romans-Clarkson SE, Walton VA, Herbison GP, et al. Psychiatric morbidity among women in urban and rural New Zealand: psycho-social correlates. Br J Psychiatry 1990;156:84-91.

17 Blazer D, George LK, Landerman R, et al. Psychiatric disorders: rural/urban comparison. Arch Gen Psychiatry 1985;42:651-6.

18 Jones K, Bullen N. Contextual models of urban house prices: a comparison of fixed-and random-coefficient models developed by expansion. Economic Geography 1994;70:252-72.

19 Goldstein H. Multilevel statistical models. London: Edward Arnold, 1995.

20 Diez-Roux AV. Multilevel analysis in public health research. Annu Rev Public Health 2000;21:171-92.

21 Snijders TAB, Bosker RJ. Multilevel analysis: an introduction to basic and advanced multilevel modeling. London: Sage, 1999.

22 Weich S, Lewis G. Material standard of living, social class and the prevalence of the common mental disorders in Great Britain. J Epidemiol Community Health 1998;52:8-14.

23 Chandola T, Bartley $M$, Wiggins $R$, et al. Social inequalities in health by individual and household measures of social position in a cohort of healthy people. J Epidemiol Community Health (in press).

24 Taylor MF, Brice J, Buck N, et al. British Household Panel Survey user manual. Vol A: Introduction, technical report and appendices. Colchester: University of Essex, 1995.

25 Goldberg DP, Williams P. The user's guide to the General Health Questionnaire. Windsor: NFER-Nelson, 1988

26 Stansfeld SA, Marmot MG. Social class and minor psychiatric disorder in civil servants: a validated screening survey using the General Health Questionnaire. Psychol Med 1992;22:739-49.

27 Goldberg D, Huxley P. Common mental disorders: a bio-social model. London: Routledge, 1992

28 Banks MH. Validation of the General Health Questionnaire in a young community sample. Psychol Med 1983;13:349-53.

29 Morris R, Carstairs V. Which deprivation? A comparison of selected deprivation indices. J Public Health Med 1991;13:318-26.

30 Wallace M, Denham C. ONS classification of local and health authorities of Great Britain. London: HMSO, 1996.

31 Simpson S, Dorling D. Those missing millions: implications for social statistics of non-response to the 1991 Census. Journal of Social Policy 1994;23:543-67.

32 Office for National Statistics. ONS classification of wards in Great Britain. London: ONS, 1998.

33 Bailey S, Charlton J, Dollamore G, et al. The ONS classification of local and health authorities. London: Office for National Statistics, 1999.

34 Jones K, Duncan C. Individuals and their ecologies: analysing the geography of chronic illness within a multilevel modelling framework. Health and Place 1995;1:27-40.

35 Goldstein H, Rasbash J, Plewis I, et al. A user's guide to MLwiN. London: Institute of Education, University of London, 1998.

36 Gilks WR, Richardson S, Spiegelhalter DJ. Markov chain Monte Carlo in practice. London: Chapman and Hall, 1996.

37 Blazer DG, Kessler RC, McGonagle KA, et al. The prevalence and distribution of maior depression in a national community sample: the National Comorbidity Survey. Am J Psychiatry 1994;151:979-86.

38 Newman SC, Bland RC, Orn H. A comparison of methods of scoring the General Health Questionnaire. Compr Psychiatry 1988;29:402-8.

39 Green AE. Unemployment, nonemployment, and labour market disadvantage. Environment and Planning A 2001;33:1361-4.

40 Burrows R, Bradshaw J. Evidence-based policies and practice. Environment and Planning A 2001;33:1345-8.

41 Birtchnell J, Masters N, Deahl M. Depression and the physical environment: a study of young married women on a London housing estate. Br J Psychiatry 1988;153:56-64.

42 Halpern D. Mental health and the built environment. London: Taylor and Francis, 1995. 
43 Sooman A Maclntyre S. Health and perceptions of the local environment in socially contrasting neighbourhoods in Glasgow. Health and Place 1995;1:15-26

44 Weich S, Blanchard $M$, Prince $M$, et al. Mental health and the built environment: a cross-sectional survey of individual and contextual risk factors for depression. Br J Psychiatry 2002;180:428-33.

45 Maclntyre S. The Black Report and beyond: what are the issues? Soc Sci Med 1997;44:723-45.

46 Ecob R, Maclntyre S. Small area variations in health related behaviours; do these depend on the behaviour itself, its measurement, or on personal characteristics? Health and Place 2000:6:261-74.

47 Davey Smith G, Whitley E, Dorling D, et al. Area based measures of social and economic circumstances: cause specific mortality patterns depend on the choice of index. J Epidemiol Community Health 2001;55:149-50.
48 Dorling D. Anecdote is the singular of data. Environment and Planning A 2001;33:1335-40.

49 Smith G, Noble $M$, Wright $G$. Do we care about area effects? Environment and Planning A 2001;33:1341-4.

50 Cohen D, Spear S, Scribner R, et al. "Broken windows" and the risk of gonorrhea. Am J Public Health 2000;90:230-6.

51 Berkman LF, Syme SL. Social networks, host resistance and mortality: a nine-year follow-up study of Alameda County residents. Am J Epidemiol 1979;109: 186-204.

52 Congdon P. Suicide and parasuicide in London: a small-area study. Urban Studies 1996:33:137-58.

53 Kawachi I, Colditz GA, Ascherio A, et al. A prospective study of social networks in relation to total mortality and cardiovascular disease in men in the US. J Epidemiol Community Health 1999;50:245-51.

\section{Clinical Evidence-Call for contributors}

Clinical Evidence is a regularly updated evidence based journal available worldwide both as a paper version and on the internet. Clinical Evidence needs to recruit a number of new contributors. Contributors are health care professionals or epidemiologists with experience in evidence based medicine and the ability to write in a concise and structured way.

Currently, we are interested in finding contributors with an interest in the following clinical areas:

Altitude sickness; Autism; Basal cell carcinoma; Breast feeding; Carbon monoxide poisoning; Cervical cancer; Cystic fibrosis; Ectopic pregnancy; Grief/bereavement; Halitosis; Hodgkins disease; Infectious mononucleosis (glandular fever); Kidney stones; Malignant melanoma (metastatic); Mesothelioma; Myeloma; Ovarian cyst; Pancreatitis (acute); Pancreatitis (chronic); Polymyalgia rheumatica; Post-partum haemorrhage; Pulmonary embolism; Recurrent miscarriage; Repetitive strain injury; Scoliosis; Seasonal affective disorder; Squint; Systemic lupus erythematosus; Testicular cancer; Varicocele; Viral meningitis; Vitiligo

However, we are always looking for others, so do not let this list discourage you.

Being a contributor involves:

- Appraising the results of literature searches (performed by our Information Specialists) to identify high quality evidence for inclusion in the journal.

- Writing to a highly structured template (about 2000-3000 words), using evidence from selected studies, within 6-8 weeks of receiving the literature search results.

- Working with Clinical Evidence Editors to ensure that the text meets rigorous epidemiological and style standards.

- Updating the text every eight months to incorporate new evidence.

- Expanding the topic to include new questions once every 12-18 months.

If you would like to become a contributor for Clinical Evidence or require more information about what this involves please send your contact details and a copy of your CV, clearly stating the clinical area you are interested in, to Claire Folkes (cfolkes@bmigroup.com).

\section{Call for peer reviewers}

Clinical Evidence also needs to recruit a number of new peer reviewers specifically with an interest in the clinical areas stated above, and also others related to general practice. Peer reviewers are health care professionals or epidemiologists with experience in evidence based medicine. As a peer reviewer you would be asked for your views on the clinical relevance, validity, and accessibility of specific topics within the journal, and their usefulness to the intended audience (international generalists and health care professionals, possibly with limited statistical knowledge). Topics are usually 2000-3000 words in length and we would ask you to review between 2-5 topics per year. The peer review process takes place throughout the year, and our turnaround time for each review is ideally 10-14 days.

If you are interested in becoming a peer reviewer for Clinical Evidence, please complete the peer review questionnaire at www.clinicalevidence.com or contact Claire Folkes (cfolkes@bmigroup.com). 